\title{
Detection of Pleural Fluid Involvement by Flow Cytometry Method in a Patient with Acute Myeloid Leukemia
}

\begin{abstract}
Keklik $M^{1 *}$, Kalan $\mathbf{U}^{2}$, Camlica $\mathrm{D}^{3}$ and Keklik $\mathrm{E}^{4}$ ${ }^{1}$ Department of Hematology, Kayseri Education and Research Hospital, Turkey

${ }^{2}$ Department of Internal Medicine, Kayseri Education and Research Hospital, Turkey

${ }^{3}$ Flow Cytometry Unit, Erciyes University, Turkey ${ }^{4}$ Department of Physiology, Erciyes University, Turkey

*Corresponding author: Muzaffer Keklik, Department of Hematology, Kayseri Education and Research Hospital, Turkey
\end{abstract}

Received: December 16, 2016; Accepted: J anuary 24, 2017; Published: January 27, 2017

\begin{abstract}
Acute leukaemias rarely present with or develop pleural effusion during their clinical course. Flow Cytometry (FC) is a potentially useful diagnostic tool for clinical practice. We report a case in which pleural effusion of an acute myeloid leukaemia (AML) was confirmed as pleural fluid involvement by FC method. A 27-years old female presented with fever and shortness of breath. On thorax CT, pleural effusion was detected in both hemithorax. Peripheral blood and pleural fluid FC was performed: CD4 (+), HLA DR (+), CD34 (+), CD33 (+), CD117 $(+)$, CD38 (+), CD11b $(+)$ and MPO $(+)$ were found and these findings were considered as consistent with AML and pleural involvement. Flow cytometry is a potentially useful diagnostic tool for clinical practice and it is a convenient method which has an important role in detection of AML in patients with pleural effusion.
\end{abstract}

Keywords: Acute myeloid leukemia; Flow cytometry; Pleural effusion

\section{Introduction}

Acute myeloid leukemia (AML) is a hematologic malignancy that carries a poor prognosis in adults. Pleural effusion in patients with AML is a rare occurrence. After a thorough search of the literature, only few cases/research articles have been found [1-3]. Recently, flow cytometry (FC) has increasingly become important in the diagnosis, prognostication and follow-up of AML [4-6]. In this study, we presented an adult AML case, in which pleural fluid involvement was detected by FC evaluation.

\section{Case Presentation}

A 27-years old female presented to emergency department with fever and shortness of breath lasting for 3-5 days. She was conscious with vital signs as follows: body temperature $38.3^{\circ} \mathrm{C}$, heart rate 100 beats per minute; blood pressure 110/60 $\mathrm{mmHg}$. Breath sounds were bilaterally fainted at basal regions of lung. On chest radiography, pleural effusion was observed. On thorax CT, pleural fluid was detected in both hemithorax (Figure 1). The following findings were detected in peripheral blood analysis: hemoglobin $(\mathrm{Hb}): 11.4 \mathrm{~g} / \mathrm{L}$ (13-16 g/L); white blood cells (WBC): 76x10 $/ \mathrm{L},\left(5.8-10.8 \times 10^{9} / \mathrm{L}\right)$;

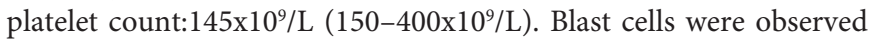
in the peripheral blood smear. A pleural tap was performed, which revealed serofibrinous fluid with a protein level of $6.2 \mathrm{~g} / \mathrm{dL}, \mathrm{Hb}: 0 \mathrm{~g} /$ L, red blood cell: 0.01 cells/lL, and WBC: $5.5 \times 10^{\%} / \mathrm{L}$. FC evaluation of peripheral blood and pleural fluid was performed by using FACS Calibur flow cytometer (Becton-Dickinson, Erembodegem, Belgium). CD4 (+), HLA DR (+), CD34 (+), CD33 (+), CD117 (+), $\mathrm{CD} 38(+), \mathrm{CD} 11 \mathrm{~b}(+)$ and MPO $(+)$ were detected in myeloid series. CD4:CD8 ratio was 38:66. Figure 2 shows these findings of FC in the pleural fluid. These findings were considered as consistent with pleural involvement of AML. Galactomannan, tuberculosis and viral evaluations of pleural fluid were found to be negative. Also, bacterial and mycobacterial culture tests were reported as negative. Diagnosis of AML was confirmed on bone marrow aspiration. Shortness of breath was relieved in patient after removing $300 \mathrm{cc}$ fluids by pleural tap. For the patient a parenteral induction chemotherapy protocol consisting of cytarabine and daunorubicin was scheduled. However, the patient developed progression of disease and she died of sepsis on the $20^{\text {th }}$ hospital day.

\section{Discussion}

Acute leukaemias rarely present with or are accompanied by pleural fluid involvement. Possible causes of pleural effusion include extramedullary proliferation of a quiescent leukaemic clone with metastasis to the bone marrow, infections, other solid tumours, and complication of chemotherapy [7]. In our case, resistant or progressive disease might be the possible aetiology. In some patients, the pleural effusion responds to treatment, whereas the resistant or the relapsing patients may necessitate a pleurodesis $[8,9]$. However, according to the literature, presence of pleural effusion may be a poor

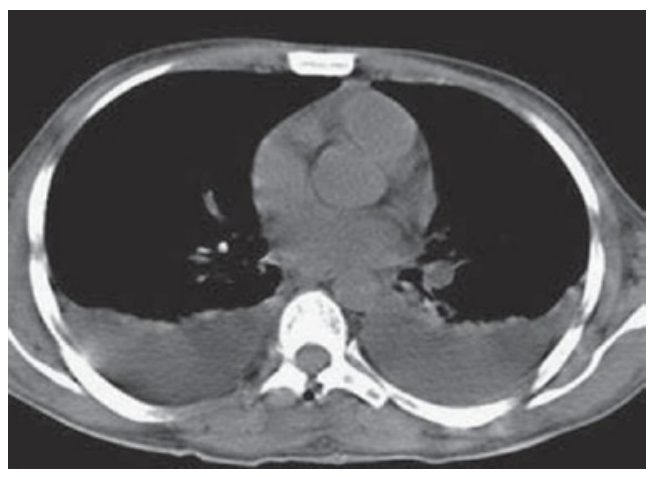

Figure 1: Axial section of a CT of the thorax showing bilateral pleural effusion.
Ann Hematol Oncol - Volume 4 Issue 1 - 2017

ISSN : 2375-7965 | www.austinpublishing group.com

Keklik et al. ( ) All rights are reserved
Citation: Keklik M, Kalan U, Camlica D and Keklik E. Detection of Pleural Fluid Involvement by Flow Cytometry Method in a Patient with Acute Myeloid Leukemia. Ann Hematol Oncol. 2017; 4(1): 1128 

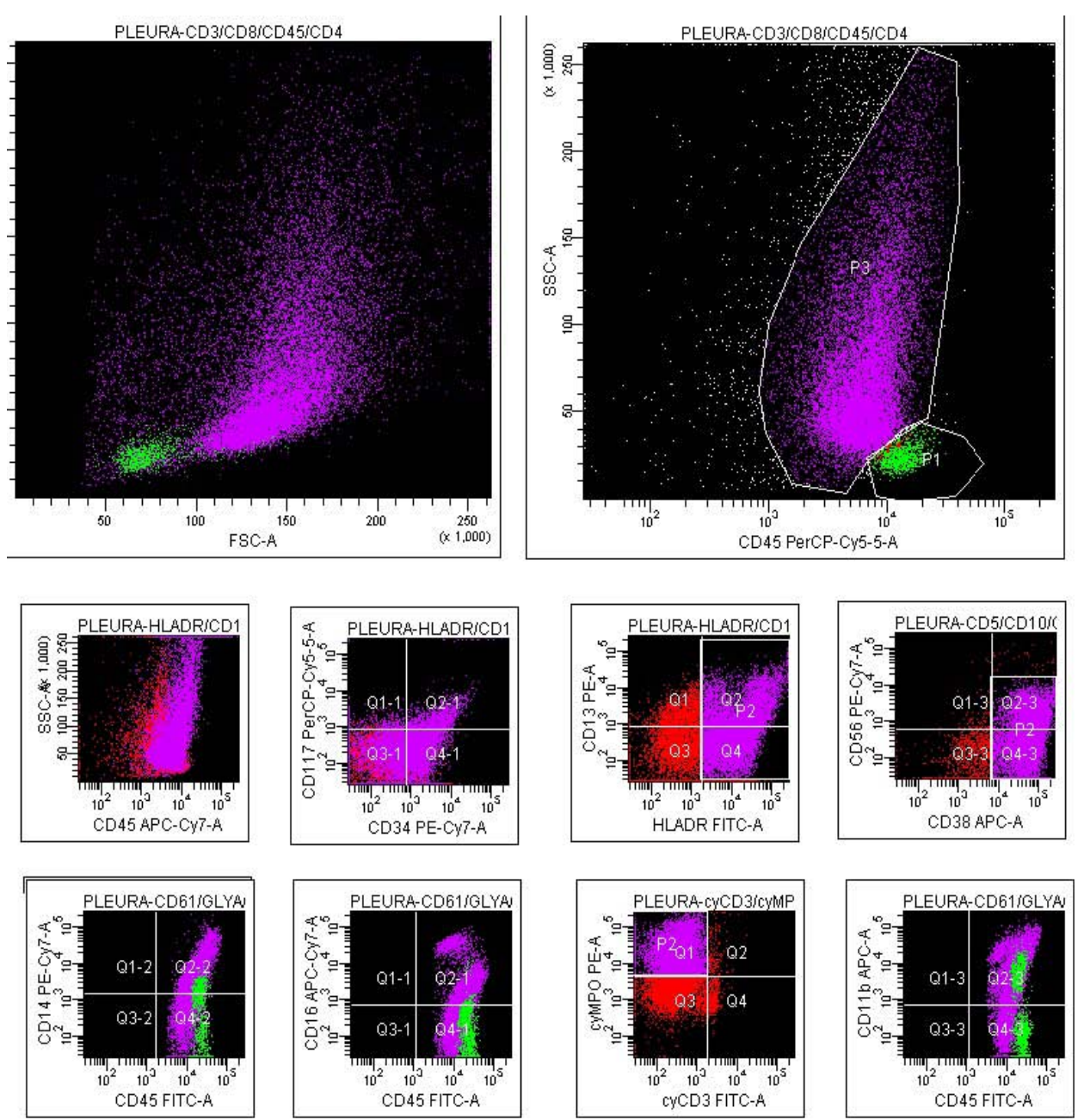

Figure 2: Findings of flowcytometry in pleural fluid.

prognostic factor, as most cases sooner succumb to disease [10-12]. Likewise our patient died a short time due to the progression. The clinician should be alert for any signs of pleural effusion in AML patients during the treatment.

Flow cytometry is an effective method for the characterization of malignant cells in effusion specimens in both the diagnostic and research settings, and this method is comparable to immunohistochemistry in terms of sensitivity and specificity, with the additional advantage of providing quantitative data [13-15]. In our case, AML diagnosis and pleural involvement were supported by FC analysis in both peripheral blood and pleural fluid.

In conclusion, in leukaemic patients, FC is frequently used as an ancillary technique for the diagnosis of hematological malignancies and it has become the most important method for diagnosis of AML. Also, it is a method than can contribute in management of malignancies as it is widely available, which can be used in analysis of all other body fluids as well as peripheral blood and bone marrow.

\section{References}

1. Raina S, Kaul R, Mahesh DM, Kaushal SS, Gupta D, Sharma J. Pleural effusion in acute myeloid leukemia: J Assoc Physicians India. 2008; 56: $914-$ 915 .
2. Duhan A, Kalra R, Kamra HT, Agarwal A, Rana P, Agarwal R, et al. Leukaemic pleural effusion as a manifestation of acute myeloid leukaemia: a case report and review of literature. Ecancermedicalscience. 2014; 8: 397.

3. Faiz SA, Bashoura L, Lei X, Sampat KR, Brown TC, Eapen GA, et al. Pleural effusions in patients with acute leukemia and myelodysplastic syndrome. Leuk Lymphoma. 2013; 54: 329-335.

4. Ommen HB. Monitoring minimal residual disease in acute myeloid leukaemia: a review of the current evolving strategies. Ther Adv Hematol. 2016; 7: 3-16.

5. Roug AS, Hansen MC, Nederby L, Hokland P. Diagnosing and following adult patients with acute myeloid leukaemia in the genomic age. $\mathrm{Br} \mathrm{J}$ Haematol. 2014; 167: 162-176.

6. Czader M, Ali SZ. Flow cytometry as an adjunct to cytomorphologic analysis of serous effusions. Diagn Cytopathol. 2003; 29: 74-78.

7. Alexandrakis MG, Passam FH, Kyriakou DS, Bouros D. Pleural effusions in hematologic malignancies. Chest. 2004; 125: 1546-1555.

8. Agarwal M, Purohit AH, Mahapatra M, Kumar R, Mishra P, Seth T, et al. Pleural effusion as an unusual initial presentation of acute myeloid leukemia. Indian J Hematol Blood Transfus. 2014; 30: 195-196.

9. Suharti C, Santosa, Setiawan B. Malignant pleural effusion in acute myeloid leukemia with hepatitis B virus infection. Acta Med Indones. 2015; 47: 153156.

10. Nieves-Nieves J, Hernandez-Vazquez L, Boodoosingh D, FernandezGonzalez R, Fernandez-Medero R, Adorno-Fontanez J, et al. Pleural effusion 
as the initial extramedullary manifestation of Acute Myeloid Leukemia. F1000Res. 1000; 1: 1-39.

11. Agrawal R. Acute Myeloid Leukaemia (AML) Presenting as a Bilateral Pleura Effusion. J Clin Diagn Res. 2013; 7: 187.

12. Chang $H$. Acute myeloid leukemia with leukemic pleural effusion. Diagn Cytopathol. 2013; 41: 909-913.

13. Iqbal J, Liu T, Mapow B, Swami VK, Hou JS. Importance of flow cytometric analysis of serous effusions in the diagnosis of hematopoietic neoplasms in patients with prior hematopoietic malignancies. Anal Quant Cytol Histol. 2010; 32: 161-165.

14. Davidson B, Dong HP, Holth A, Berner A, Risberg B. Flow cytometric immunophenotyping of cancer cells in effusion specimens: diagnostic and research applications. Diagn Cytopathol. 2007; 35: 568-578.

15. Kalina T, Flores-Montero J, van der Velden VH, Martin-Ayuso M, Bottcher $\mathrm{S}$, Ritgen $\mathrm{M}$, et al. EuroFlow standardization of flow cytometer instrument settings and immunophenotyping protocols. Leukemia. 2012; 26: 1986-2010.
Ann Hematol Oncol - Volume 4 Issue 1 - 2017

ISSN : 2375-7965 | www.austinpublishinggroup.com

Keklik et al. (C) All rights are reserved
Citation: Keklik M, Kalan U, Camlica D and Keklik E. Detection of Pleural Fluid Involvement by Flow Cytometry Method in a Patient with Acute Myeloid Leukemia. Ann Hematol Oncol. 2017; 4(1): 1128. 